
Argonne National Laboratory ("Argonne") under Contract No. W-31-109-ENG-38 with the U.S. Department of Energy. The U.S. Government retains for itself, and others act. ing on its behalf, a paid-up, nonexclusive, irrevocable worldwide llcense in said article to reproduce, prepare derivative works. dis. tribute copies to the public, and pertorm publicly and display publicly, by or on behall of the Government.

\title{
CONTEMPORARY APPLICATIONS OF DYSON-SCHWINGER EQUATIONS
}

\author{
M. B. HECHT, C. D. ROBERTS and S. M. SCHMIDT \\ Physics Division, Bldg. 203, Argonne National Laboratory \\ Argonne IL 60439-4843, USA
}

\begin{abstract}
We illustrate the contemporary application of Dyson-Schwinger equations using two examples: the calculation of pseudoscalar meson masses, an associated modelindependent mass formula and the approach to the heavy-quark limit; and the study of nucleon observables, including a calculation of its mass via a covariant Fadde'ev equation and an estimate of pion-loop contributions to this mass.
\end{abstract}

1. Introductory Remarks. Just in case anyone remains uninformed, the Dyson-Schwinger equations (DSEs) are an analogue in quantum field theory of the Euler-Lagrange equations in classical field theory. They are an enumerable infinity of coupled integral equations whose solutions are the $n$-point Schwinger functions (Euclidean Green functions). These Schwinger functions are also the matrix elements estimated in numerical simulations of lattice-QCD. The coupling between equations is a nuisance, of course: it necessitates a truncation in order to define a tractable problem. A weak coupling expansion provides one systematic method and reproduces perturbation theory. However, it also makes nonperturbative phenomena inaccessible, and something else is needed for the study of strongly interacting systems and bound state phenomena.

This is a situation familiar from many body theory, in which case the Hartree-Fock truncation often yields reliable information. There is an analogue in QCD: the renormalisation-group-improved rainbow-ladder truncation, which is phenomenologically efficacious, as is clear from Ref. [1] wherein the power of a single-parameter model of the infrared behaviour of the effective quark-quark interaction is illustrated. (The ultraviolet behaviour is fixed and model-independent because the DSEs reproduce perturbation theory.) The successes and failures of such a model can be understood once it is appreciated that the rainbow-ladder truncation is the leading order in a systematic, Ward-Takahashi identity preserving $1 / N_{c}$-like expansion. ${ }^{2}$ But this demonstration is just one small step toward a rigorous foundation for contemporary DSE modelling.

One of the beauties of a model is that its simplicity makes possible a rapid comparison between theory and experiment. Another is that it can be wrong: an Ansatz is intuitively motivated and explored, and, if it is widely successful, its failures can point to unanticipated phenomena. Irrespective therefore of the 


\section{DISCLAIMER}

This report was prepared as an account of work sponsored by an agency of the United States Government. Neither the United States Government nor any agency thereof, nor any of their employees, make any warranty, express or implied, or assumes any legal liability or responsibility for the accuracy, completeness, or usefulness of any information, apparatus, product, or process disclosed, or represents that its use would not infringe privately owned rights. Reference herein to any specific commercial product, process, or service by trade name, trademark, manufacturer, or otherwise does not necessarily constitute or imply its endorsement, recommendation, or favoring by the United States Government or any agency thereof. The views and opinions of authors expressed herein do not necessarily state or reflect those of the United States Government or any agency thereof. 


\section{DISCLAIMER}

Portions of this document may be illegible in electronic image products. Images are produced from the best available original document. 


\section{3. \\ NOV $0 \& 2000$ \\ OSTI}

difficulties that remain in providing a rigorous foundation for the application of DSEs in QCD, their phenomenological application plays an important part in attempts to elucidate nonperturbative phenomena.

This is nowhere clearer than in the study of dynamical chiral symmetry breaking (DCSB). The DSEs provide the simplest and most direct means of understanding the dichotomy of the pion as both a Goldstone mode and a bound state of a massive dressed-quark and -antiquark, ${ }^{3,4}$ and the result is model-independent. The analysis relies on the interplay between the QCD gap equation, the DSE for the dressed-quark propagator, and the inhomogeneous Bethe-Salpeter equation (BSE) for the axial-vector vertex. This interplay is a consequence only of the axial-vector Ward-Takahashi identity. Furthermore, a key quantitative conclusion follows: ${ }^{5,6}$ in order to reproduce observed characteristics of the spectrum, the kernel in the QCD gap equation must exhibit a significant enhancement on the domain $\Lambda_{\mathrm{QCD}}^{2} \lesssim k^{2} \lesssim 2 \mathrm{GeV}^{2}$. Identifying the origin of that enhancement; i.e., whether it is a feature of the dressed-gluon propagator alone or of the contraction of this propagator with the dressedquark-gluon vertex, is currently an important focus. ${ }^{6,7,8}$

In the last decade the use of DSEs in QCD has attracted renewed interest and they have been applied to a broad range of phenomena, as is clear from the detailed summaries in Refs. $[9,10]$. The approach has moved far beyond the calculation of indigent approximations to $f_{\pi}$ and the vacuum quark condensate. Herein we illustrate this by focusing on two topics: pseudoscalar meson masses and their evolution with the current-quark mass; and a description of the nucleon, its interactions and the calculation of its mass.

2. Pseudoscalar Meson Masses. Meson masses can be calculated by solving the renormalised homogeneous BSE:

$$
\left[\Gamma_{H}(k ; P)\right]_{t u}=\int_{q}^{\Lambda}\left[\chi_{H}(q ; P)\right]_{s r} K_{t u}^{r s}(q, k ; P),
$$

where: $r, \ldots, u$ represent colour-, Dirac- and flavour-matrix indices and $H$ identifies the meson under consideration; $P$ is the total momentum and $P^{2}=-m_{H}^{2}$ is the eigenvalue condition for a solution; $\chi_{H}(q ; P):=\mathcal{S}\left(q_{+}\right) \Gamma_{H}(q ; P) \mathcal{S}\left(q_{-}\right)$is the Bethe-Salpeter wave function, with $\Gamma_{H}(q ; P)$ the fully-amputated BetheSalpeter amplitude and $\mathcal{S}=\operatorname{diag}\left(S_{u}, S_{d}, S_{s}, \ldots\right)$ the dressed-quark propagator flavour matrix, $q_{+}=q+\eta_{P} P, q_{-}=q-\left(1-\eta_{P}\right) P^{a}$ and $K(q, k ; P)$ is the renormalised, fully-amputated quark-antiquark scattering kernel, which is

${ }^{a} \eta_{P} \in[0,1]$ is the momentum partitioning parameter. It appears because in a Poincaré covariant approach the definition of the relative momentum is arbitrary. Observable quantities must be independent of $\eta_{P}$. 
two-particle-irreducible, with respect to the quark-antiquark pair of lines, and does not contain quark-antiquark to single gauge-boson annihilation diagrams, such as would describe the leptonic decay of a pseudoscalar meson. In Eq. (1) $\int_{q}^{\Lambda}:=\int^{\Lambda} d^{4} q /(2 \pi)^{4}$ represents mnemonically a translationally-invariant regularisation of the integral, with $\Lambda$ the regularisation mass-scale, and in this particular case the r.h.s. of the renormalised equation is cutoff-independent. $(\Lambda \rightarrow \infty$ is the final step in all calculations.)

For a pseudoscalar meson the solution of Eq. (1) has the form

$$
\begin{aligned}
\Gamma_{H}(q ; P)= & T^{H} \gamma_{5}\left[i E_{H}(q ; P)+\gamma \cdot P F_{H}(q ; P)\right. \\
& \left.+\gamma \cdot q q \cdot P G_{H}(q ; P)+\sigma_{\mu \nu} q_{\mu} P_{\nu} H_{H}(q ; P)\right],
\end{aligned}
$$

where $T^{H}$ is the flavour matrix that specifies the mesonic channel under consideration; e.g., $T^{K^{+}}=(1 / \sqrt{ } 2)\left(\lambda^{4}+i \lambda^{5}\right)$, with $\left\{\lambda^{i}, i=1, \ldots, 8\right\}$ the Gell-Mann matrices. The amplitude is canonically normalised by requiring that the bound state contribution to the fully-amputated quark-antiquark scattering matrix: $. M=K+K(\mathcal{S S}) K+\ldots$, have unit residue and the constraint that ensures this is given; e.g., in Ref. [4].

Equation (1) is the equation satisfied by the residue of the meson pole in the inhomogeneous Bethe-Salpeter equation. Using: the inhomogeneous Bethe-Salpeter equations for the axial-vector and pseudovector vertices; the dressed-quark DSE; and the fact that a nonperturbative Ward-Takahashi identity preserving truncation of the DSEs is possible, it was shown in Ref. [3] that, for flavour nonsinglet pseudoscalar mesons,

$$
f_{H} m_{H}^{2}=\mathcal{M}_{H}^{\zeta} r_{H}^{\varsigma},
$$

with $\mathcal{M}_{H}^{\zeta}:=\operatorname{tr}_{\text {flavour }}\left[M_{(\zeta)}\left\{T^{H},\left(T^{H}\right)^{t}\right\}\right]$, where $M_{(\zeta)}=\operatorname{diag}\left(m_{\mathrm{u}}^{\zeta}, m_{d}^{\zeta}, m_{s}^{\zeta}, \ldots\right)$ and $(\cdot)^{t}$ indicates matrix transpose, so that $\mathcal{M}_{H}^{\zeta}$ is proportional to the sum of the constituents' current-quark masses. This is a model-independent identity, which is valid for all current-quark masses, irrespective of their magnitude, and therefore provides a single formula that unifies both the light- and heavy-quark regimes.

In Eq. (3), $f_{H}$ is the leptonic decay constant, which the derivation proves is given by

$$
f_{H} P_{\mu}=Z_{2} \int_{q}^{\Lambda} \frac{1}{2} \operatorname{tr}\left[\left(T^{H}\right)^{\mathrm{t}} \gamma_{5} \gamma_{\mu} \chi_{H}(q ; P)\right],
$$

where $Z_{2}=Z_{2}(\zeta, \Lambda)$ is the dressed-quark wave function renormalisation constant, with $\zeta$ the renormalisation point. This multiplicative factor of $Z_{2}$ on 
the r.h.s. ensures that $f_{H}$ is gauge-invariant, and independent of the renormalisation point and regularisation mass scale; i.e., that it is an observable. ${ }^{3}$ Equation (4) yields $f_{H}$ as the pseudovector projection of the meson's BetheSalpeter wave function at the origin in configuration space; i.e., this equation provides a field theoretical analogue of the "wave function at the origin," which describes the decay of bound states in quantum mechanics.

The remaining term in Eq. (3) is

$$
i r_{H}^{\zeta}=Z_{4} \int_{q}^{\Lambda} \frac{1}{2} \operatorname{tr}\left[\left(T^{H}\right)^{t} \gamma_{5} \chi_{H}(q ; P)\right],
$$

where $Z_{4}=Z_{4}(\zeta, \Lambda)$ is the dressed-quark mass renormalisation constant. The gauge dependence of $Z_{4}$ is just that necessary to ensure that the r.h.s. of Eq. (3) is gauge invariant; its cutoff dependence ensures that the r.h.s. is independent of the cutoff; and its renormalisation point dependence ensures that the product on the r.h.s. is independent of the renormalisation point. ${ }^{3} \tau_{H}^{\zeta}$ is the pseudoscalar projection of the meson's Bethe-Salpeter wave function at the origin in configuration space.

In asymptotically free theories the chiral limit is unambiguously defined ${ }^{3,4}$ by setting $\hat{m}=0$, where $\hat{m}$ is the renormalisation point independent currentquark mass. In this limit ${ }^{3}$

$$
r_{H_{0}}^{\zeta}:=\lim _{\bar{m} \rightarrow 0} r_{H}^{\zeta}=-\frac{1}{f_{H_{0}}}\langle\bar{q} q\rangle_{\zeta}^{0},
$$

where $f_{H_{0}}$ is obtained by taking the chiral limit in Eq. (4) and the vacuum quark condensate is

$$
-\langle\bar{q} q\rangle_{\zeta}^{0}=Z_{4} N_{c} \int_{q}^{\Lambda} \operatorname{tr}_{D}\left[S_{\hat{m}=0}(q)\right],
$$

with $S_{\dot{m}=0}$ obtained as the chiral limit solution of the dressed-quark DSE: ${ }^{b}$ this is the gauge-invariant and cutoff-independent expression for the condensate. Using Eq. (6), Eq. (3) yields

$$
f_{H_{0}}^{2} m_{H}^{2}=-\left(m_{f_{1}}^{\zeta}+m_{f_{2}}^{\zeta}\right)\langle\bar{q} q\rangle_{\zeta}^{0}+O\left(\hat{m}_{f_{1}, f_{2}}^{2}\right),
$$

with $f_{1,2}$ labelling the flavour of the dressed-quark constituents; i.e., as a corollary Eq. (3) yields what is commonly known as the Gell-Mann-Oakes-Renner relation.

\footnotetext{
${ }^{b}$ See, e.g., Eq. (1) of Ref. [1] with $m \rightarrow 0$ on the r.h.s.
} 
As remarked above, Eq. (3) is also valid for arbitrarily large current-quark masses and an analysis of its heavy-quark limit is facilitated by writing $P_{\mu}=$ $m_{H} v_{\mu}=\left(\hat{M}_{Q}+E_{H}\right)$, where $\hat{M}_{Q}$ is a constituent-heavy-quark mass ${ }^{11}$ and $E_{H}$ is a "binding energy." Following this the dressed-heavy-quark propagator takes the form

$$
S_{Q}(k+P)=\frac{1}{2} \frac{1-i \gamma \cdot v}{k \cdot v-E_{H}}+\mathrm{O}\left(\frac{|k|}{\hat{M}_{Q}}, \frac{E_{H}}{\hat{M}_{Q}}\right),
$$

where $k$ is the momentum of the lighter constituent, and the canonically normalised Bethe-Salpeter amplitude can be expressed as

$$
\Gamma_{H}(k ; P)=\sqrt{m_{H}} \Gamma_{H}^{<\infty}(k ; P),
$$

where $\Gamma_{H}^{<\infty}(k ; P)$ is pointwise finite in the limit $m_{H} \rightarrow \infty$. Using Eqs. (9) and (10) in Eq. (4) yields ${ }^{11}$

$$
f_{H}=\frac{c_{H}^{f}}{\sqrt{m_{H}}}
$$

with $c_{H}^{f}$ a calculable and finite constant, which reproduces a well-known consequence of heavy-quark symmetry. Applying the same analysis to Eq. (5) one finds ${ }^{12}$

$$
r_{H}=c_{H}^{r_{\zeta}} \sqrt{m_{H}}
$$

and this, along with Eq. (11) in Eq. (3), proves ${ }^{12,13}$ that in the heavy-quark limit

$$
m_{H}=\frac{c_{H}^{r_{c}}}{c_{H}^{f}} \mathcal{M}_{H}^{\zeta}
$$

i.e., that the mass of a heavy pseudoscalar meson rises linearly with the mass of its heaviest constituent.

In Ref. [13] it was shown that Eq. (11) is not valid until current-quark masses $m \gtrsim m_{b}$. The $c$-quark lies well-outside this domain; e.g., if a constant of proportionality is chosen so as to reproduce the value of $f_{B}$, then $f_{D}$ obtained from this formula is $\sim 40 \%$ too large. This is consistent with the calculated magnitude ${ }^{13}$ of the violations of heavy-quark symmetry in $b \rightarrow c$ transitions $(\lesssim 30 \%)$.

Using the results reported in Ref. [1], obtained using the renormalisationgroup-improved rainbow-ladder truncation of Ref. [14], one can study the evolution of meson masses as the current-quark mass is increased. Consider first pseudoscalar mesons whose constituents have equal current-masses, for which the calculated evolution is described by the interpolating formula 1

$$
m_{H^{*}}=1.04 \sqrt{\mathcal{X}}+0.21 \mathcal{X}
$$


where $\mathcal{X}=m^{\zeta} / \Lambda_{\mathrm{QCD}}$, with $\zeta=19 \mathrm{GeV}$ and $\Lambda_{\mathrm{QCD}}=0.234$. This formula was determined via an unconstrained fit to the masses calculated by solving the Bethe-Salpeter equation. A comparison with Eq. (8) shows that $B_{0}^{\zeta}:=\beta^{2} /\left(2 \Lambda_{\mathrm{QCD}}\right)=-\langle\bar{q} q\rangle_{\zeta}^{0} / f_{H_{0}}^{2}$ and, using the model's calculated value of $f_{H_{0}}=0.088 \mathrm{GeV}$, one infers a value of $\langle\bar{q} q\rangle_{\zeta}^{0}=(-0.26 \mathrm{GeV})^{3}$ from this correspondence. That can be compared with the value $(-0.27)^{3}$ calculated directly in this model from Eq. (7). This near equality indicates that the interpolating formula in Eq. (14) can provide reliable estimates.

Equation (14) indicates that a flavour nonsinglet, $f_{1}=f=f_{2}$ pseudoscalar meson with a mass $m_{H}=1 \mathrm{GeV}$ would be composed of quarks with mass $\hat{m}_{f}=0.32 \Lambda_{\mathrm{QCD}}=2.3 \hat{m}_{s}$. At this current-quark mass, which corresponds to $\mathcal{X}=0.68$, the $\sqrt{\mathcal{X}}$ term still provides $86 \%$ of the meson's mass. Thus one is still well away from the linear trajectory, in spite of the fact that in the neighbourhood of $\mathcal{X}=0.68$ a tangent to the curve in the left panel of Fig. 2, Ref. [I], is nearly indistinguishable from the curve itself within the resolution of that figure. Furthermore one finds easily from Eq. (14) that

$$
\frac{m_{H_{m_{f}=2 m_{s}}^{\pi}}^{2}}{m_{H_{m_{f}=m_{s}}^{*}}^{2}}=2.2
$$

in agreement with the result obtained in recent lattice simulations. ${ }^{15}$ Thus, in addition to being phenomenologically effrcacious, the renormalisation-groupimproved rainbow-ladder truncation of Ref. [14] predicts a mass-evolution that is confirmed by lattice simulations. These results support a scenario of dynamical chiral symmetry breaking in which the vacuum quark condensate is large; i.e., $B_{0}^{1 \mathrm{GeV}} \gg f_{H_{0}}$.

Reference [1] also provides an interpolation of the kaon-like $u$ - $q$ trajectory:

$$
m_{H^{K}}=0.083+0.5 \sqrt{\mathcal{X}}+0.31 \mathcal{X}
$$

and while the BSE studies reviewed in Ref. [14] have not directly addressed heavy-light nor heavy-heavy bound states (systems with $\hat{m}_{f}>3.5 \hat{m}_{s}$ have not been studied) one may, as a preliminary step, ask whether Eq. (16) can be used to obtain reliable mass-estimates via extrapolation? Using Eq. (16), one reproduces $m_{D} \simeq 1.9 \mathrm{GeV}, m_{B} \simeq 5.3 \mathrm{GeV}$ with $m_{c}^{1 \mathrm{GeV}} \simeq 1.1 \mathrm{GeV}, m_{b}^{1 \mathrm{GeV}} \simeq$ $4.2 \mathrm{GeV}$, and since these current-quark masses are in agreement with other estimates $^{16}$ then Eq. (16) can be a useful tool. In this application one finds that the linear term provides $50 \%$ of $m_{D}$ and $67 \%$ of $m_{B}$. Thus, like the conclusion drawn on the validity of Eq. (11) for $f_{H}$, the heavy-quark limit in Eq. (13) is not valid until current-quark masses satisfy $m \gtrsim m_{b}$, and the $c$-quark lies well below this lower limit. 
3. A Model of the Nucleon. The success of the rainbow-ladder truncation in describing meson observables motivates ${ }^{17}$ a treatment of the nucleon as a bound state of a dressed-quark and nonpointlike diquark via a covariant Fadde'ev equation. The feasibility of this approach was demonstrated in Ref. [18] and the most extensive study to date is described in Ref. [19]. The approach assumes only that the colour- $\overline{3}$ quark-quark scattering matrix can be approximated by a sum of diquark pseudoparticle terms: scalar + pseudovector $+\ldots$, whose properties can be determined independently. The Fadde'ev equation then describes the nucleon as a quark-diquark composite, which is bound by the repeated exchange of roles between the dormant and diquarkparticipant quarks, and the complete nucleon amplitude is a sum of three terms:

$$
\Psi=\Psi_{1}+\Psi_{2}+\Psi_{3},
$$

where the subscript identifies the dormant quark and; e.g., $\Psi_{1,2}$ are obtained from $\Psi_{3}$ via a cyclic permutation of all the indices: $\Psi_{1}=\Psi_{3}\left(\downarrow_{231}^{123}\right), \Psi_{2}=$ $\Psi_{3}\left(\downarrow_{312}^{123}\right)$.

The simplest such model retains only the contribution of the scalar diquark to the quark-quark scattering matrix, in which case

$$
\begin{aligned}
& \Psi_{3}\left(p_{i} ; \alpha_{i}, \tau_{i} ; \alpha, \tau\right)= \\
& \epsilon_{c_{1} c_{2} c_{3}} \delta^{\tau \tau_{3}} \Delta^{0^{+}}(K)\left[\Gamma_{0^{+}}\left(\frac{1}{2} p_{[12]} ; K\right)\right]_{\alpha_{1} \alpha_{2}}^{\tau_{1} \tau_{2}} \psi_{3}(\ell ; P) u(P),
\end{aligned}
$$

where: $(i \gamma \cdot P+M) u(p)=0$, with $P=p_{1}+p_{2}+p_{3}=: p_{\{123\}}$ the nucleon's total momentum and $M$ its mass; $\epsilon_{c_{1} c_{2} c_{3}}$ is the colour-singlet factor; $K=p_{1}+p_{2}=: p_{\{12\}}, p_{[12]}:=p_{1}-p_{2}, \ell=\left(p_{3}-p_{\{12\}}\right) / 2 ;\left(\alpha_{i}, \tau_{i}\right)$ are the quark spinor and isospin labels, and $(\alpha, \tau)$ are those of the nucleon. In Eq. (18), $\Delta^{0^{+}}$is the pseudoparticle propagator for a scalar diquark formed from quarks 1 and 2 , and $\Gamma_{0^{+}}$is a Bethe-Salpeter-like amplitude describing their relative momentum correlation. Both these quantities are determined by studying the quark-quark scattering matrix. The remaining element, $\psi_{3}$, is a Bethe-Salpeter-like amplitude that describes the relative momentum correlation between the third quark and the diquark's centre-of-momentum. It satisfies a renormalised Fadde'ev equation, which in the isospin symmetric limit assumes the form

$$
\begin{aligned}
\psi_{3}(k ; P)= & -2 \int_{\ell}^{\Lambda} \Delta^{0^{+}}\left(K_{\ell}\right) \Gamma_{0^{+}}(k+\ell / 2 ; K) \\
& \times S\left(\ell_{\mathrm{ex}}\right)^{\mathrm{t}} \bar{\Gamma}_{0^{+}}\left(\ell+k / 2 ;-K_{k}\right) S\left(\ell_{1}\right) \psi_{3}(\ell ; P),
\end{aligned}
$$

with $K_{\ell}=-\ell+(2 / 3) P, \ell_{\mathrm{ex}}=-\ell-k-P / 3, \ell_{1}=\ell+P / 3$. The general solution 
for a positive energy nucleon takes the form

$$
\psi_{3}(\ell ; P)=f_{1}(\ell ; P) \mathbf{1}-\frac{1}{M}(i \gamma \cdot \ell-\ell \cdot \hat{P} \mathbf{1}) f_{2}(\ell ; P),
$$

with $\hat{P}^{2}=-1$ and where $f_{1,2}$ describe, respectively, the upper/lower component of the dressed-nucleon spinor.

The nucleon amplitude in Eqs. (17), (18) has been used with success to calculate a wide range of leptonic and nonleptonic nucleon form factors. ${ }^{20-22}$ In those calculations solving the Fadde'ev equation was side-stepped by employing the following simple parametrisations for the functions that appear in Eqs. (18), (20):

$$
\begin{aligned}
\Delta^{0^{+}}\left(K^{2}\right) & =\frac{1}{m_{0^{+}}^{2}} \mathcal{F}\left(K^{2} / \omega_{0^{+}}^{2}\right), \\
\Gamma_{0^{+}}(k ; K) & =\frac{1}{\mathcal{N}_{0^{+}}} C i \gamma_{5} i \tau_{2} \mathcal{F}\left(k^{2} / \omega_{0^{+}}^{2}\right), \\
f_{1}(\ell ; P) & =\frac{1}{\mathcal{N}_{\psi}} \mathcal{F}\left(\ell^{2} / \omega_{\psi}^{2}\right),
\end{aligned}
$$

with $f_{2}=R f_{1}$, where $\mathrm{R}$ is a constant of proportionality that gauges the relative importance of the lower component of the nucleon spinor. In these equations $C=\gamma_{2} \gamma_{4}$ is the charge conjugation matrix, $\mathcal{F}(y)=\left(1-\mathrm{e}^{-y}\right) / y$, and $\mathcal{N}_{\psi}, \mathcal{N}_{0+}$ are calculated, canonical normalisation constants.

The parameters in this model are $\omega_{0+}, m_{0^{+}}, \omega_{\psi}: d_{0^{+}}=1 / \omega_{0+}$ measures the quark-quark separation in the diquark; $\ell_{0^{+}}=1 / m_{0^{+}}$is the diquark correlation length or mean free path; and $d_{\psi}=1 / \omega_{\psi}$ measures the quark-diquark separation. ${ }^{c}$ Their values have been determined by requiring a good impulseapproximation fit to the proton's charge form factor on $Q^{2} \in[0,3] \mathrm{GeV}^{2}$ and this procedure yields

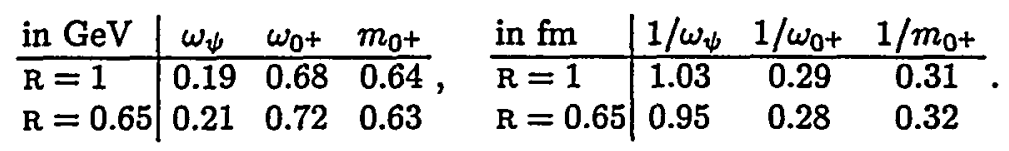

The scalar diquark parameter values determined in these unconstrained fits are within $10 \%$ of those obtained in the BSE studies of Ref. [23].

\footnotetext{
${ }^{c}$ A description of the nucleon in this form can only be internally consistent if $d_{0+}<d_{\psi}$ and $\ell_{0+}<d_{\psi}$; $i$.e., the diquark is smaller than the nucleon and can't propagate over distances larger than the nucleon.
} 


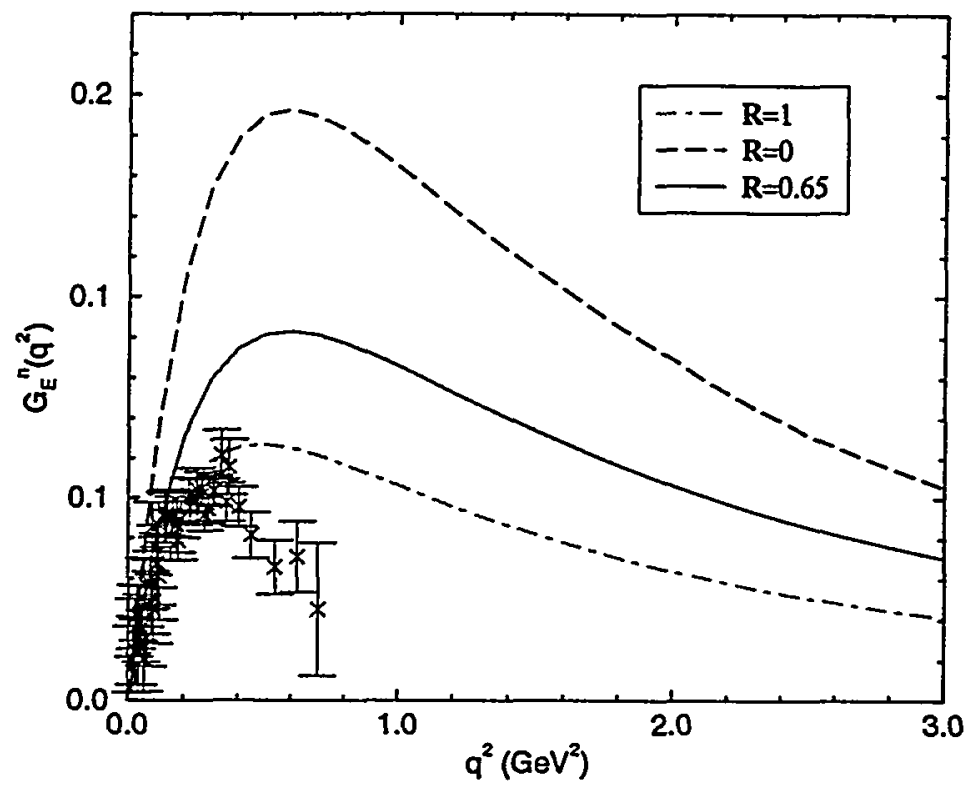

Figure 1: Neutron electric form factor calculated with three different values of $\mathrm{R}$. Data from Ref. [24], extracted using the Argonne V18 potential. ${ }^{25} G_{E}^{n}$ is very sensitive to the strength of $f_{2}$. (NB. The contribution of the pseudovector diquark was not included in these calculations.)

Using the values in Eq. (24) a wide range of observables can be calculated and herein we exemplify the results via

\begin{tabular}{l|ccccccc} 
& $r_{p}^{2}\left(\mathrm{fm}^{2}\right)$ & $r_{n}^{2}\left(\mathrm{fm}^{2}\right)$ & $\mu_{p}\left(\mu_{N}\right)$ & $\mu_{n}\left(\mu_{N}\right)$ & $\mu_{n} / \mu_{p} g_{\pi N N}$ & $g_{A}$ \\
\hline Emp. & $(0.87)^{2}$ & $-(0.34)^{2}$ & 2.79 & -1.91 & -0.68 & 13.4 & 1.26 \\
\hline Calc. $\mathrm{R}=1$ & $(0.78)^{2}$ & $-(0.34)^{2}$ & 2.82 & -1.62 & -0.57 & 14.6 & 1.27 \\
Calc. $\mathrm{R}=0.65$ & $(0.81)^{2}$ & $-(0.37)^{2}$ & 2.85 & -1.63 & -0.57 & 14.5 & 1.12
\end{tabular}

and the neutron electric form factor in Fig. 1. One significant feature, apparent in Eq. (25), is that $\left|\mu_{n} / \mu_{p}\right|>0.5$, which is only possible because the impulse approximation explicitly includes diquark breakup contributions. Another is a prediction for the ratio $\mu_{p} G_{E}^{p}\left(q^{2}\right) / G_{M}^{p}\left(q^{2}\right)$ that is in semi-quantitative agreement with recent results from TJNAF. ${ }^{26}$ As shown explicitly in Ref. [9], the impulse approximation requires five terms when the diquark is composite, a fact also appreciated in Ref. [27]. It also requires an explicit form for the dressed-quark propagator but that is well-known from studies of meson observables and is given; e.g., in Refs. [20,21]. 


\begin{tabular}{c|cccc|cccc} 
& $\omega_{0+}$ & $m_{0^{+}}$ & $\omega_{1+}$ & $m_{1+}$ & $\mathrm{R}$ & $\omega_{\psi_{1}}$ & $\omega_{\psi_{2}}$ & $M$ \\
\hline $0^{+}$ & 0.68 & 0.64 & - & - & 1.11 & 0.40 & 0.43 & 1.48 \\
$0^{+} \& 1^{+}$ & 0.68 & 0.64 & 0.68 & 0.82 & 0.62 & 0.38 & 0.41 & 1.28 \\
$0^{+} \& 1^{+}$ & 0.68 & 0.64 & 0.40 & 0.82 & 0.73 & 0.32 & 0.35 & 1.16 \\
$0^{+} \& 1^{+}$ & 0.40 & 0.64 & 0.40 & 0.82 & 1.11 & 0.29 & 0.31 & 1.14 \\
$0^{+} \& 1^{+}$ & 0.68 & 0.54 & 0.40 & 0.69 & 0.54 & 0.31 & 0.36 & 0.94
\end{tabular}

Table 1: Nucleon mass and $\mathrm{R}=f_{2} / f_{1}$ ratio determined by solving the Fadde'ev equation. $\omega_{\psi_{1}}, \omega_{\psi_{f_{2}}}$ are the widths of a least-squares fit to $f_{1}, f_{2}$ assuming they are pointwise well-approximated by $\mathcal{F}\left(\ell^{2} / \omega_{\psi_{f}}\right)$. (They actually fall faster with increasing $k^{2}$ but these widths are nevertheless useful for comparison with Eq. (25).) The first row gives the results obtained with the scalar diquark alone while the others were obtained with the inclusion of a pseudovector diquark correlation. All dimensioned quantities are given in $\mathrm{GeV}$.

Hitherto the choice of $\mathbf{R}$ is arbitrary. However, its value is fixed by solving the Fadde'ev equation. To exemplify that we have solved the Fadde'ev equation, Eq. (19), with the values of $\omega_{0+}, m_{0+}$ in Eq. (24) and assuming that $f_{i}(\ell ; P)=f_{i}\left(\ell^{2}\right)$. This yields the results in Table 1 . As anticipated in Ref. [21], a reduction of $1 / 3$ is required in the calculated mass for agreement with experiment and here the inclusion of a pseudovector diquark can help. ${ }^{19}$

To explore that we added such a correlation to Eq. (18):

$$
\psi_{3}^{1^{+}}=\epsilon_{c_{1} c_{2} c_{3}} \Delta_{\mu \nu}^{1^{+}}(K) i \gamma_{\mu} C{T^{i}}^{i} \Gamma_{1+}\left(\frac{1}{2} p_{[12]}\right) \mathcal{A}_{\nu}^{i}(\ell ; P) u(P)
$$

where: $\left\{\mathrm{T}_{i=(+, 0,-)}=\left(\tau_{0}+\tau_{3}\right) / \sqrt{2}, \tau_{1},\left(\tau_{0}-\tau_{3}\right) / \sqrt{2}\right\}, \tau_{0}=\operatorname{diag}(1,1)$, are the symmetric isospin-triplet matrices,

$$
\mathcal{A}_{\nu}^{i}(\ell ; P)=a_{1}^{i}\left(\ell^{2}\right) \gamma_{5} \gamma_{\nu}+a_{2}^{i}\left(\ell^{2}\right) \gamma_{5} \gamma \cdot \hat{\ell} \hat{\ell}_{\nu}, \hat{\ell}^{2}=1,
$$

with $a_{1}^{1}=a_{1}^{2}=a_{1}^{3}, a_{2}^{1}=a_{2}^{2}=a_{2}^{3}$ in the isospin symmetric limit; and

$$
\begin{aligned}
\Delta_{\mu \nu}^{i^{+}}(K) & =\left(\delta_{\mu \nu}+\frac{K_{\mu} K_{\nu}}{m_{1+}^{2}}\right) \frac{1}{m_{1+}^{2}} \mathcal{F}\left(K^{2} / \omega_{1+}^{2}\right), \\
\Gamma_{1+}\left(k^{2}\right) & =\frac{1}{\mathcal{N}_{1+}} \mathcal{F}\left(k^{2} / \omega_{1+}^{2}\right) ;
\end{aligned}
$$

and extended Eq. (19) to include the coupling to this channel.

The results obtained in this case are also presented in Table 1. In the calculations we chose the value of $m_{1+} / m_{0+}$ from Ref. [23] and, initially, $\omega_{1+}=$ $\omega_{0+}$, to find, as anticipated, that the pseudovector diquark provides additional attraction and reduces the calculated mass by $11 \%$. Reducing $\omega_{1+}$ increases the 
pseudovector diquark coupling via an increase in $1 / \mathcal{N}_{1+}$, hence the calculated mass is reduced: a $40 \%$ reduction in $\omega_{1}+$ reduces $M$ by $10 \%$. Reducing $\omega_{0+}$ by the same amount has very little effect, reducing $M$ by only an extra $2 \%$. However, in agreement with intuition, decreasing the diquark masses reduces the calculated nucleon mass: a $21 \%$ decrease yields a $19 \%$ reduction in $M$. It is clear from the table that an internally consistent description of the nucleon is possible using only scalar and pseudovector diquark correlations, just as found in Ref. [19]. Furthermore, it is clear from the table that a well-constrained scalar diquark model should employ $R$ in the range $0.5-0.7$.

A question that remains unaddressed is the role of pion loops. The $\pi N N$ coupling is strong and hence it is conceivable that such loops might generate large self energy corrections to the nucleon's mass. We have made an estimate using a model DSE for the nucleon self energy: ${ }^{d}$

$$
G^{-1}(p)=i \gamma \cdot p+M+3 \int \frac{d^{4} k}{(2 \pi)^{4}} \Delta_{\pi}(p-k) g_{\pi N N}^{2}\left((p-k)^{2}, k^{2}\right) \gamma_{5} G(k) \gamma_{5},
$$

where $\Delta_{\pi}(\ell)=1 /\left(\ell^{2}+m_{\pi}^{2}\right)$ and $g_{\pi N N}\left((p-k)^{2}, k^{2}\right)$ is the momentum-dependent $\pi N N$ coupling. The $t=-(p-k)^{2}$-dependence of this coupling was calculated for on-shell nucleons in Ref. [21], with the result

$$
g_{\pi N N}\left(t, k^{2}=-M^{2}\right) \approx \frac{g_{\pi N N}}{\left(1-t / \Lambda_{\pi}^{2}\right)^{2}}, \Lambda_{\pi}=0.96 \mathrm{GeV}
$$

where $g_{\pi N N}$ is given in Eq. (25). This is not quite sufficient for our present purpose because the nucleon in the loop is not on-shell. Therefore to complete an estimate we employ a simple product Ansatz:

$$
g_{\pi N N}(p ; k)=\frac{g_{\pi N N}}{\left(1+\left|p^{2}+k^{2}\right| / \Lambda_{\pi}^{2}\right)^{2}\left(1+\left(\left|p^{2}+M^{2}\right|+\left|k^{2}+M^{2}\right|\right) / \Lambda_{\pi}^{2}\right)^{2}},
$$

to approximate the angular-average of the coupling, which is active in the integral equation. With the parameters in the last row of Table 1 we find that the pion loop adds $10 \mathrm{MeV}$ to the nucleon's mass; i.e., it provides only a $1 \%$ increase. The detailed form of Eq. (31) is not important but the off-shell suppression is.

4. Epilogue. Two short summaries is all we have room for here. In large part, the light-quark meson sector is well understood. The renormalisation-groupimproved rainbow-ladder truncation provides reliable information in many channels and where it doesn't the reasons why are understood. ${ }^{2}$ Understanding the nucleon: it's mass and interactions, is. a contemporary focus and

\footnotetext{
${ }^{d}$ The positive sign on the r.h.s. is correct and entails that the mass shift is positive.
} 
progress is rapid. Success there will open many new phenomenological applications, such as the calculation of quark distribution functions, which are merely parametrised in analyses of deep inelastic scattering. That the DSEs can provide valuable insight here is demonstrated by a calculation of the valence quark distribution in the pion, ${ }^{28}$ which itself is measurable given a high-luminosity electron-proton collider. ${ }^{29}$

Acknowledgments. CDR is grateful for the hospitality and support of the Erwin Schrödinger Institute for Mathematical Physics, Vienna, which helped to make possible his participation in this workshop, and to the organisers of Quark Confinement and the Hadron Spectrum IV; SMS is grateful for financial support from the A. v. Humboldt foundation; and we acknowledge useful communications with J.C.R. Bloch and P. Maris. This work was supported by the US Department of Energy, Nuclear Physics Division, under contract no. W-31-109-ENG-38, the National Science Foundation under grant no. INT9603385 , and benefited from the resources of the National Energy Research Scientific Computing Center.

\section{References}

1. P. Maris, "Continuum QCD and Light Mesons," this volume.

2. A. Bender, C.D. Roberts and L. v. Smekal, Phys. Lett. B 380 (1996) 7.

3. P. Maris, C.D. Roberts and P.C. Tandy, Phys. Lett. B 420 (1998) 267.

4. P. Maris and C.D. Roberts, Phys. Rev. C 56 (1997) 3369.

5. F.T. Hawes, P. Maris and C.D. Roberts, Phys. Lett. B 440 (1998) 353.

6. C.D. Roberts, "Continuum Strong QCD: Confinement and Dynamical Chiral Symmetry Breaking," nucl-th/0007054.

7. L. v. Smekal and R. Alkofer, "What the Infrared Behavior of QCD Green Functions can tell us about Confinement in the Covariant Gauge," this volume.

8. A.G. Williams, "Lattice Studies of Confinement and Chiral Symmetry Breaking in a Covariant Gauge," this volume.

9. C.D. Roberts and S.M. Schmidt, "Dyson-Schwinger equations: Density, temperature and continuum strong QCD," nucl-th/0005064, to appear in Prog. Part. Nucl. Phys. 45 (2000).

10. R. Alkofer and L. v Smekal, "The infrared behavior of QCD Green's functions: Confinement, dynamical symmetry breaking, and hadrons as relativistic bound states," hep-ph/0007355.

11. M.A. Ivanov, Yu.L. Kalinovsky, P. Maris and C.D. Roberts, Phys. Lett. B 416 (1998) 29. 
12. P. Maris and C.D. Roberts, "QCD bound states and their response to extremes of temperature and density," in Proc. of the Workshop on Nonperturbative Methods in Quantum Field Theory, edited by A.W. Schreiber, A.G. Williams and A.W. Thomas (World Scientific, Singapore, 1998) pp. 132-151.

13. M.A. Ivanov, Yu.L. Kalinovsky and C.D. Roberts, Phys. Rev. D 60 (1999) 034018.

14. P. Maris and P.C. Tandy, Phys. Rev. C 60 (1999) 055214.

15. K. C. Bowler et al. [UKQCD Collaboration], Phys. Rev. D 62 (2000) 054506.

16. Particle Data Group (D.E. Groom et al.), Eur. Phys. J. C 15, 1 (2000).

17. R.T. Cahill, C.D. Roberts and J. Praschifka, Austral. J. Phys. 42 (1989) 129.

18. C.J. Burden, R.T. Cahill and J. Praschifka, Austral. J. Phys. 42 (1989) 147.

19. G. Hellstern, R. Alkofer, M. Oettel and H. Reinhardt, Nucl. Phys. A 627 (1997) 679.

20. J.C.R. Bloch, C.D. Roberts, S.M. Schmidt, A. Bender and M.R. Frank, Phys. Rev. C 60 (1999) 062201.

21. J.C.R. Bloch, C.D. Roberts and S.M. Schmidt, Phys. Rev. C 61 (2000) 065207.

22. M.B. Hecht, C.D. Roberts and S.M. Schmidt, "DSE Hadron Phenomenology," nucl-th/0005067.

23. C.J. Burden, L. Qian, C.D. Roberts, P.C. Tandy and M.J. Thomson, Phys. Rev. C 55 (1997) 2649.

24. S. Platchkov et al., Nucl. Phys. A 510 (1990) 740.

25. R.B. Wiringa, private communication; R.B. Wiringa, V.G. Stoks and R. Schiavilla, Phys. Rev. C 51 (1995) 38.

26. M.K. Jones et al. [Jefferson Lab Hall A Collaboration], Phys. Rev. Lett., 84, 1398 (2000).

27. M. Oettel, M. Pichowsky and L. v Smekal, Eur. Phys. J. A 8 (2000) 251.

28. M.B. Hecht, C.D. Roberts and S.M. Schmidt, "Valence-quark distributions in the pion," nucl-th/0008049.

29. R.J. Holt and P.E. Reimer, "Structure of the Goldstone Bosons," ANL-preprint. 